\title{
Graphite and Interstellar Extinction ${ }^{1}$
}

\author{
T. P. Stecher and Bertram DonN \\ NASA Goddard Space Flight Center \\ Greenbelt, Maryland
}

THE THEORY THAT GRAPHITE PARTICLES could be responsible for interstellar extinction is presented in references 1 and 2 , and this paper will present evidence that further supports this contention. The complex dielectric constant $(m=n(1-i k))$ for graphite has been measured as a function of energy by the authors of reference 3 , and the optical constants as function of wavelength $\lambda$ have been obtained from this measurement. A large variation occurs in both $n$ and $k$ at wavelengths below $3000 \AA$.

The results reported in the preceding paper indicated the desirability of performing Mie scattering calculations on graphite. The measured values of the complex index of refraction corresponding to 17 wavelengths were used in the calculation for spherical particles. The IBM 7094 computer program for Mie scattering follows that of van de Hulst. (See ref. 4.) The Oort-van de Hulst size distribution (ref. 5), which may not be applicable to the type of particle discussed herein, has been used to obtain an integrated cross section for extinction. The steps were in terms of $0.8 \times 10^{-6} \mathrm{~cm}$ for the particle radius with the $1 /$ eth value of the frequency function being $5.6 \times 10^{-6} \mathrm{~cm}$. In figure 1 , this curve is compared with the mean observed interstellar extinction of Boggess and Borgman (ref. 6) and the further observational results reported in the preceding paper. The maximum in the theoretical extinction curve at $\lambda^{-1}=4.4$ microns $^{-1}$ is the signature of graphite. This maximum rapidly increases as the relative size of the particle radius decreases. At the same time, the extinction for $\lambda^{-1}<2$ decreases. A 50 -percent increase in the relative radius of the particles appears sufficient to produce the variation in the ratio of selective to total extinction observed by the author of reference 7 . A maximum in the observed interstellar extinction curve also occurs at $\lambda^{-1}=4.4$ microns $^{-1}$. The

\footnotetext{
1 The contents of this paper were published previously in the Astrophys. J. (pub. by the Univ. of Chicago Press), vol. 142, 1965, p. 1681.
} 


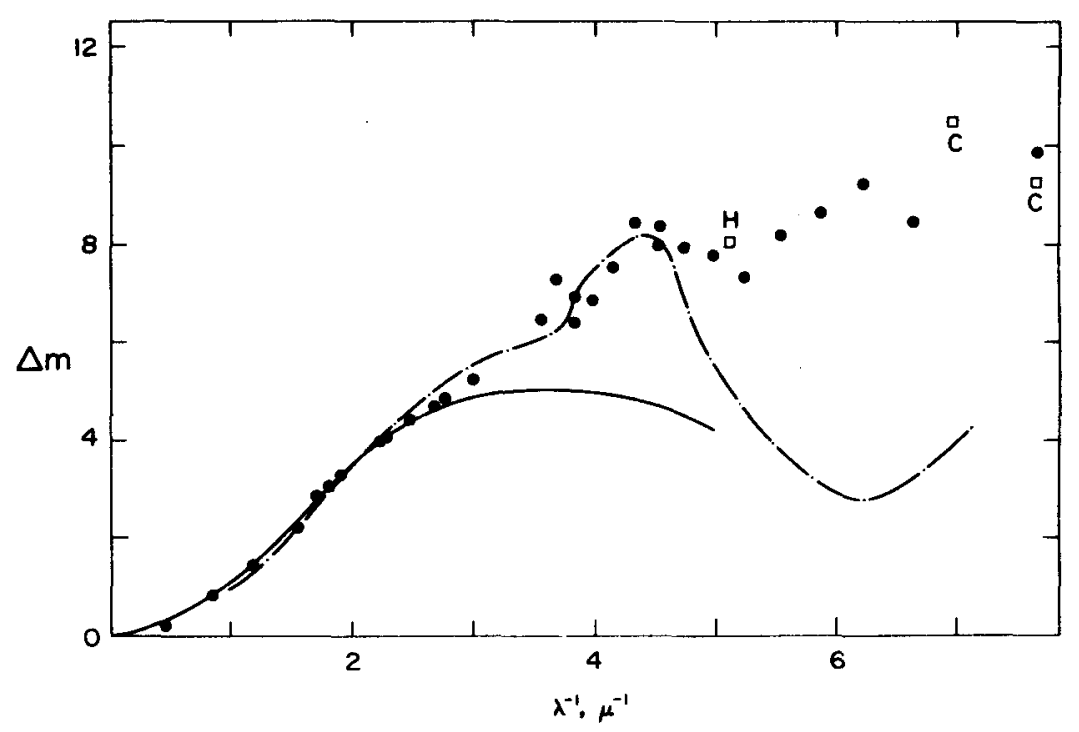

FIGURE 1. - Observed and theoretical interstellar extinction. Circles for $\lambda^{-1}>3 \mu^{-1}$ : mear. observed interstellar extinction reported in paper by Stecher in the present compilation; circles for $\lambda^{-1} \leqslant 3 \mu^{-1}$ : mean observed extinction of investigation of ref. 6; squares marked $H$ and $C$ : observed extinction obtained from other sources as reported in the paper by Stecher in the present compilation; dash-dot curve: theoretical interstellar extinction for graphite grains; solid curve: theoretical extinction by van de Hulst (ref. 5).

authors think that this coincidence provides a strong argument in favor of graphite. For the first time there is structure in the extinction curve and it can be accounted for by a particular substance. It is, of course, possible that some other material could have the same signature and also be abundant in interstellar space, but this seems improbable. The albedo for this particle-size distribution is somewhat larger than that reported in reference 8 and may be, sufficiently high to account for reflection nebulae (ref. 9).

For shorter wavelengths, the calculated curve is inconsistent with the observations. It seems unlikely that a particle-size distribution could be found that would be satisfactory. The addition of a dielectric material either as a coating of the graphite or as separate small particles could easily bring up the curve. A promising "dielectric" which is already present is graphite itself. Graphite is strongly anisotropic. The calculations of the present investigation were made with optical constants measured for the electric vector in the basal planes. When the electric vector is perpendicular to the basal planes, the conductivity is at least 100 times smaller (ref. 8) and graphite then acts like a dielectric. Since graphite is presumed to be present in the form of flakes that are almost randomly oriented, a sizable proportion of the flakes will present this thin dielectric face to the radiation. 
The fact that both the ratio of crystal axis and the "dielectric" index of refraction are uncertain precludes a quantitative calculation at this time. This conjecture could be observationally checked by polarization measurements at $\lambda^{-1}=6 \mu^{-1}$. If graphite is assumed to be the sole cause of interstellar extinction, a reversal in the sign of polarization would be expected when the "dielectric" extinction exceeds that of the conducting plane.

In addition to the proposal of reference 2 for interstellar carbon grains, it has been pointed out (ref. 10) that graphite flakes may grow in space if countering effects can be neglected. The exponential whisker growth mechanism (ref. 11) was shown to apply. For a plate of $10^{-6} \mathrm{~cm}$ thickness, the length reaches $5 \times 10^{-6} \mathrm{~cm}$ in $6 \times 10^{8}$ years for $N_{\mathrm{H}}=1 / \mathrm{cm}^{3}$ and in $6 \times 10^{7}$ years for the number density of hydrogen atoms $N_{\mathrm{H}}=10 / \mathrm{cm}^{3}$.

Grains such as these are intermediate in structure and mass between classical grains proposed by van de Hulst (ref. 5) and Platt particles (ref. 12).

The assumption that the grains are graphite indicates the possibility of obtaining considerable detailed information concerning the particlesize distribution in the interstellar clouds between the Earth and any particular star by means of rocket observations.

\section{REFERENCES}

1. Cayrel, P.; and Schatzman, E.: Sur la Polarisation Interstellaire par des Particules de Graphite. Ann. Astrophys., vol. 17, 1954, p. 555.

2. Hoyle, F.; and Wickramasinghe, N. C.: On Graphite Particles as Interstellar Grains. Roy. Astron. Soc., Monthly Notices, vol. 124, 1962, p. 417.

3. Taft, E. A.; and Philipp, H. R.: Optical Properties of Graphite. Phys. Rev., vol. 138A, 1965, p. 197.

4. VAN DE Hulst, H. C.: Light Scattering by Small Particles. John Wiley \& Sons, Inc., 1957.

5. Van de Hulst, H. C.: The Solid Particles in Interstellar Space. Rech. Astron. Obs. Utrecht, vol. 11, pt. 2, 1949.

6. BogGess, A., III; and Borgman, J.: Interstellar Extinction in the Middle Ultraviolet. Astrophys. J., vol, 140, 1964, p. 1636.

7. Johnson, H. L.: Interstellar Extinction in the Galaxy. Astrophys. J., vol. 141, 1965, p. 923.

8. Wickramasinghe, N. C.: A Note on Interstellar Polarization by Graphite Flakes. Roy. Astron. Soc., Monthly Notices, vol. 125, 1962, p. 87.

9. Van Houten, C. J.: Surface Photometry of Extragalactic Nebulae. Bull. Astron. Inst. Netherlands, vol. 16, no. 509 , 1961 , p. 1.

10. Donn, B.: The Physics and Chemistry of Interstellar Grains. I. Crystal Growth in Space Astron. J., vol. 70, 1965, p. 320.

11. Sears, G. W.: The Origin of Screw Dislocations - Role of Colloidal Particles. Acta Metallurgica, vol. 3, 1955, p. 306.

12. Platt, J. R.: On the Optical Properties of Interstellar Dust. Astrophys. J., vol. 123, 1956, p. 486. 


\section{DISCUSSION}

Greenberg: I would like to inquire about the two points in the extreme ultraviolet obtained by Chubb and Byram; can you explain how one obtains the extinction from their data?

Stecher: I looked up the $B-V$ colors for all the stars they had observed and plotted them in terms of magnitude. There are a couple of $B$ stars way off from the highly reddened end, and there is a cluster of $B$ stars presumably unreddened that have very little color excess in the $B-V$ region. I didn't attempt to put a probable error on it, as this was sort of a straight-line fit through the diagram and it was just indicative. As I mentioned, there was much scatter.

Nandy: You have used three $O$ stars in the Perseus region and two $\mathrm{O}$ stars in the Scorpius region. H. L. Johnson has recently reported that the Scorpius stars show an extinction law similar to that in Cygnus, whereas the Perseus stars exhibit a different extinction law. Can you combine your observations if a difference exists in the extinction law?

Stecher: The observations that $I$ have are weak enough so that between intrinsic variation and observational error I really don't want to say there is an intrinsic variation. The data indicate that this bump at $\lambda 2200 \AA$ is stronger in the Scorpius region. On the graphite interpretation this would mean more small particles.

Nandy: It is interesting that in the Perseus region the slope of the ultraviolet part relative to the blue is different from that in Cygnus.

Stecher: Yes, I am aware of that and I have no explanation.

Wickramasinghe: This slope difference could be due to a difference in the sizes of the particles. Smaller graphite particles mixed with larger ones would give the bump indicated.

Greenberg: You presented two curves, one of which gave a match, more or less, to some spot in the ultraviolet which is characteristic of graphite as reported in the literature. I qualify that by saying that it is not necessarily graphite, but suppose it is. That first one matched the extinction in the near ultraviolet and the visible very poorly. The next curve that you gave was presented with this mixture of particles where you got a relatively good fit from $\lambda^{-1}=0.80$ up to $\lambda^{-1}=3$. What I don't understand is how you combined these two calculations, the one which didn't fit anywhere, but which did fit, more or less, the bump in the ultraviolet, and the other which fit in the near ultraviolet down to the far infrared.

Stecher: I thought the first curve fit quite well.

Greenberg: As I recall, it deviated very drastically around $\lambda^{-1}=3$, and it deviated very significantly, $I$ believe, around $\lambda^{-1}=1.5$ to 2 . It wasn't drawn any farther down than that. The point I'm raising is what we mean by a fit. At $\lambda^{-1}=3$ this curve is considerably off from the 
observations and just seems to cross somewhere. I can always make curves cross somewhere, but I don't see that it looks anything like the observed points.

Stecher: The graphite refractive indices were obtained from a very small graph in the Physical Review and the second curve was plotted from Taft and Phillips' original numbers. Therefore, there is a slight difference, especially in the infrared, a region in which I was not particularly interested. The difference between Boggess and Borgman's points and van de Hulst's curve is about 15 percent of the scaling of the size distribution. I don't think that a 15-percent difference in the scaling of the radii is important.

Greenberg: For the graphite? I don't understand. Your graphite curve does not fit in the near ultraviolet. It doesn't really fit anywhere; the slope is all wrong. I'm raising a point which I feel is very important: What do we mean by a fit? Now if one is matching signature - there is a signature of this hump at about $\lambda^{-1}=4.4$ - that $I$ think would be rather significant if there is an absorptive type of material which we can identify. I think this is important even if we don't match it anywhere else. However, I don't believe that there is a fit to an extinction curve.

Wickramasinghe: I don't see your objection. The fit in the visible and near ultraviolet is quite satisfactory to within the observational error. The hump itself is the signature I think; this is the most interesting and significant point. Dr. Stecher used an Oort-van de Hulst distribution of sizes, although I think there is no particular justification for using it with graphite particles. I have done it with several types of distributions, mainly with Gaussian dispersions centered at $0.05 \mu$ with dispersion $\sigma$ between $0.01 \mu$ and $0.02 \mu$. One gets a very good fit in the visible as well as a hump centered at $\lambda^{-1}=2200 \AA$. It is important to note that Dr. Stecher's observations show a pronounced quenching effect centered at about $\lambda^{-1}=0.22 \mu$ for stars located in different parts of the galaxy. This fact suggests strongly that one is observing a spectroscopic feature of the grains - independent of the size.

Stecher: Since I didn't have any really strong justification for using the Oort-van de Hulst distribution, the difference in the scaling of the radii of something on the order of 10 percent will run it through a mean curve which again is not really what one should fit. This is why I didn't attempt to fit it any closer. I think the general agreement is reasonably good.

Greenberg: If you change the size to bring that curve down to fit in the near ultraviolet, the curve will drop down farther on in the ultraviolet. You will still have the hump when you bring it down, and it will have the same qualitative features. The details of the extinction in the far ultraviolet are perhaps still not known.

Wickramasinghe: Yes, one should insist, I think, on a perfectly $271-992$ O-67-2 
good fit between the theory and the observations in the visible, while also reproducing the qualitative features of the ultraviolet observations at least.

Greenberg: Yes, we do know something about the visible, but how much we know about the far infrared is another point that can be raised.

Nandy: I have a definition of a good fit. By good fitting, I mean that the variance should be less than the standard error of the observations. Therefore, it is important to know the standard error of the observations. If the standard error is very large, fitting will be poor in any case.

Wickramasinghe: Yes, $I$ think it is worth adding to this observation that the standard of error is in general greater in the ultraviolet than in the visible. And, if one draws bars denoting the rms error at each wavelength point, one should get a fit within the error bars for any tenable grain model.

Borgman: In answer to the remark of Dr. Wickramasinghe, I think that observational errors could not explain the deviation from the theoretical curve-these errors are so large that they certainly must mean that the theoretical curve or the physical variables used in the computations must be in error. 